\title{
UNLOCKING RHIZOSPHERIC BACTERIA SECONDARY METABOLISM: GENOME ANALYSIS FOR THE DISCOVERY OF NOVEL ANTIMICROBIAL COMPOUNDS
}

\author{
Daniel Espinosa-Sáiz ${ }^{1}$, Zaki Saati-Santamaría ${ }^{1,2}$, Esther Menéndez ${ }^{1,2,3}$, Pedro F. Mateos ${ }^{1,2,4}$. \\ 1 Microbiology and Genetics Department. University of Salamanca, 37007 Salamanca, Spain \\ 2 Spanish-Portuguese Institute for Agricultural Research (CIALE), Universidad de Salamanca, \\ 37185 Villamayor, Spain
}

3 Mediterranean Institute for Agriculture, Environment and Development (MED), Institute for Advanced Studies and Research (IIFA), Universidade de Évora, Pólo da Mitra, Ap. 94, 7006-554 Évora, Portugal

4 Associated RandD Unit, USAL-CSIC (IRNASA), 37008 Salamanca, Spain

\section{Abstract}

The emergence of antimicrobial resistance in pathogenic agents has raised awareness among society and nowadays is a recognized threat to public health. This problem is aggravated due to the misuse of current antibiotics and the lack of novel antimicrobial compounds ${ }^{1}$. Soil microorganisms are a potential source of new antibiotics and, thanks to the study of their genomes, we can guide the search for undescribed antimicrobial compounds ${ }^{2,3}$. We have isolated two bacterial strains from a rhizospheric soil, belonging to the genera Brevibacillus and Streptomyces, which were revealed as antimicrobial agents, inhibiting the growth of bacteria and fungi with different profiles of antimicrobial resistance. We sequenced the genome of these strains using the Illumina MiSeq platform. The gene calling and genome annotation were done through the RAST tool $(\mathrm{v} 2.0)^{4}$. antiSMASH $(\mathrm{v} 5.1)^{5}$ was used to annotate in depth those genes related to the secondary metabolism of both strains. Genome analyses showed diverse antimicrobial potential encoded within these 2 genomes. In sum, 61 biosynthetic gene clusters (BGCs) related with the secondary metabolism were annotated, of which 16 correspond to the Brevibacillus strain and 45 to the Streptomyces strain. The most abundant BGCs were nonribosomal peptide synthetase (NRPS), terpenes and siderophores. Interestingly, some of these BGCs showed no similarity to any of the already described ones involved in the production of antimicrobial compounds. Therefore, the genetic machinery encoded in both genomes might provide us the basis for the discovery of novel antibiotics against multidrug resistance pathogens.

\section{References}

1. Arias CA, Murray BE. Enterococcus species, Streptococcus gallolyticus group, and Leuconostoc species. En: Bennett JE, Dolin R, Blaser MJ, editores. Mandell, Douglas, and Bennett's Principles and Practice of Infectious Diseases. 8th ed. Philadelphia: Elsevier Saunders; 2015, p. 2328-2339

2. Ziemert $\mathrm{N}$, Alanjary $\mathrm{M}$, Weber $\mathrm{T}$. The evolution of genome mining in microbes - a review. RSC. 2016; 33: 988-1005

3. Adamek M, Alanjary M, Ziemert N. Applied evolution: phylogeny-based approaches in natural products research. RSC. 2019; 36: 1295-1312

4. Aziz RK, Bartels D, Best AA. et al. The RAST Server: Rapid Annotations using Subsystems Technology. BMC Genomics. 2008; 9: 75 
5. Blin K, Shaw S, Steinke K, Villebro R, Ziemert N, Lee SY, Medema MH, Weber T. antiSMASH 5.0: updates to the secondary metabolite genome mining pipeline. Nucleic Acids Research. 2019; 47: 81-87 